\title{
Screening for Chlamydia trachomatis
}

\section{Opportunistic approaches have little evidence to support them}

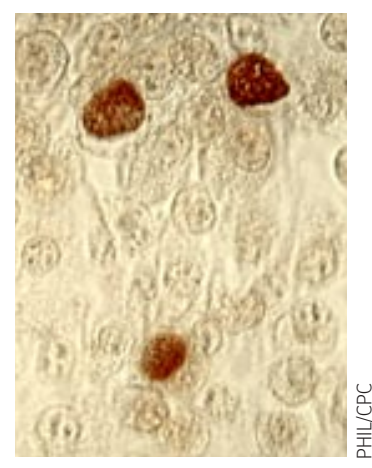

ANALYSIS p 725

Rachael Jones consultant physician

Department of HIV/Genitourinary Medicine, St Stephen's Centre,

Chelsea and Westminster NHS

Foundation Trust, London

SW10 9NH

rachael.jones@chelwest.nhs.uk

Fiona Boag consultant physician

Competing interests: None declared.

Provenance and peer review:

Commissioned; not externally peer

reviewed.

BMJ 2007;334:703-4

doi:10.1136/bmj.39167.545417.80
Sexual infections and teenage pregnancies disproportionately affect people living in poverty and social exclusion. In 2004, the government white paper "Choosing health" ${ }^{1}$ identified sexual health as a priority area for improvement and service development. The paper followed the publication of the National Strategy for Sexual Health and HIV ${ }^{2}$ which aims to provide "better prevention, better services, and better sexual health." The strategy described the implementation of a broad National Chlamydia Screening Programme (NCSP) in accordance with the National Health Service plan, which included "a commitment to improving the prevention of ill health and providing screening programmes where they are appropriate."

In this week's $B M J$, an analysis by Low shows how acceptance of the effectiveness of chlamydial screening programmes in Sweden and the United States supported the funding of the National Chlamydia Screening Programme before the balance of benefits and harms was thoroughly understood. ${ }^{3}$

Chlamydia is the most common sexually transmitted disease in England. Its prevalence has increased steadily since the mid-1990s, and rose by 300\% from 1995 to 2004. The main burden of infection affects women aged 16-19 and men aged 20-24 years. Although often asymptomatic, associated problems such as pelvic inflammatory disease, infertility, ectopic pregnancy, epididymo-orchitis, ophthalmic complications, and neonatal complications are well described. As with most sexually transmitted infections, Chlamydia trachomatis may be a cofactor for HIV transmission.

Chlamydia is the only sexually transmitted infection for which population screening has been implemented. Ideally, a screening programme for infectious disease should identify and treat a sufficient number of infections to reduce transmission in the community, and thus reduce prevalence. There are two main approaches to the design of screening programmes-proactive and opportunistic. Proactive screening-for example, cervical screening in the United Kingdom-uses the population register to identify the target population. People are contacted at intervals defined by the transmission dynamics (which use epidemiological data to estimate the spread or transmission of infection in the absence of intervention within a defined time frame), uptake of screening is monitored, and non-attenders are contacted. Opportunistic screening targets people who attend a healthcare setting. Thus, it reaches only those who attend the service and regular rescreening is unlikely to occur. It also relies upon the health provider remembering to give information and offer the test to those deemed eligible.

The National Chlamydia Screening Programme in England began in 2001. ${ }^{4}$ It uses an opportunistic approach and targets sexually active people under 25 years of age within a variety of healthcare settings. It is a dynamic model, which evolves as evidence accumulates. In the third year of the programme, 1777 venues were involved in 26 programme areas, and nearly 100000 people were screened. Most of these people were women $(82 \%)$. The incidence of chlamydia infection was $10.1 \%$ (95\% confidence interval 10.0 to 10.3$)$. Of 8816 positive cases, around 10000 partners were reported; $49 \%$ of partners were tested and $33 \%$ were treated. This may seem like a low proportion, but contact tracing is notoriously difficult. In a prior attempt to develop national standards for measuring outcomes of care for gonorrhoea and chlamydia in genitourinary medicine clinics, reports detailing chlamydia outcomes were identified. In large city clinics, 0.43 ( 0.30 to 0.62$)$ contacts per case were screened compared with 0.64 (0.58 to 0.70$)$ contacts per case in other clinics.

The analysis by Low $^{3}$ cites a lack of evidence from randomised controlled trials to support the opportunistic screening method. The opportunistic approach is used in most chlamydia screening programmes in the US, Sweden, and England. Studies showing a reduction in the rates of chlamydia, pelvic inflammatory disease, and ectopic pregnancy over time have been widely cited in support of such screening. ${ }^{5}{ }^{6}$ A concurrent decrease in the diagnosis of gonorrhoea in Sweden and the UK has occurred, but has been ascribed to extensive safe sex campaigns rather than the chlamydia screening programme. However, rates of chlamydia in Sweden, the rest of Europe, and the US have been steadily increasing since the mid-1990s despite the implementation of opportunistic screening. This increase in rates may reflect the outcomes of targeting more people, the use of more sensitive technology, or a genuine increase in prevalence.

Critics of the National Chlamydia Screening Programme have focused on several issues. These include the use of an opportunistic screening method and its associated fragility; the reduced participation of general practitioners in the programme compared with the pilot schemes, in which doctors were paid for each patient enrolled and participation was mandatory; the low number of men screened; contact tracing failing to reach all partners; and cost effectiveness. In two pilot sites, all general practitioners took part, were paid for each patient enrolled, ${ }^{5}$ and generated the highest proportion of tests and cases, achieving an effective screening rate of $50 \%{ }^{3}$ 
In the programme itself, participation of general practitioners is optional and largely unremunerated. In 2005-6, the effective screening rate was less than $5 \%$ in more than half of programme areas. However, the National Chlamydia Screening Programme is in its third phase of conception and is still expanding, with a large scale implementation due later this year. It has responded with innovative strategies, such as pharmacy based screening programmes, to reach target groups.

A proactive approach to chlamydia screening might be difficult and unacceptable to some people, as screening would start at 16 years of age and not all of those invited would be sexually active. As the aim of the programme is to reduce the prevalence of chlamydia, infected men would need to be identified, and this might have a significant impact on its cost effectiveness. Strategies from the men's health forum study (supported by the Department of Health), such as depositing test kits and health promotion literature in the workplace, may prove useful. ${ }^{7}$ Cost is being questioned by Low ${ }^{3}$ in light of conflicting opinions regarding the prevalence of morbidity related to chlamydia, and mathematical modelling relies on high levels of acceptance, uptake, and coverage of screening, in addition to annual repeat testing and partner notification-areas that are continually being developed in the
National Chlamydia Screening Programme.

The Department of Health has recruited the National Institute for Health and Clinical Excellence (NICE) to produce public health guidance on interventions, including screening, designed to reduce the transmission of chlamydial infection, together with other sexually transmitted infections and pregnancy in the under $18 \mathrm{~s} .^{8}$ Recommendations include identifying people at risk of infection and providing sexual health counselling. One to one interventions, as well as group and peer based approaches, are highlighted throughout the document. It discusses partner notification and treatment; evidence based methods that have yielded higher contact rates than conventional contact tracing, such as mailing home sampling urine kits and patient delivered partner therapy (this strategy is not legal in the UK). Primary care trust commissioners are asked to ensure that sexual health services are in place to meet local needs.

Sexual health has emerged as a government priority. Despite multiple campaigns in the media, the diagnosis of sexually transmitted infections continues to increase. Most people who are affected are unlikely to seek sexual health testing and may only be assessed via a proactive approach rather than the opportunistic screening programme currently offered.

\section{Coeliac disease in primary care}

\section{Is common, underdiagnosed, and can present with non-specific symptoms}

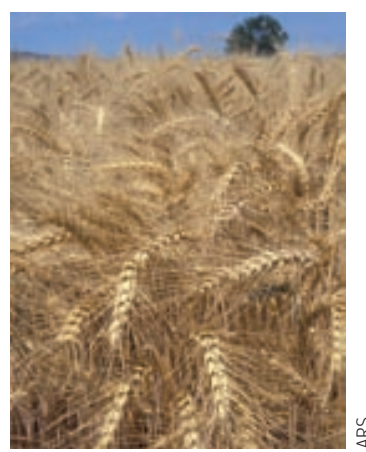

RESEARCH pp 729,732

Roger Jones Wolfson professor of general practice

Department of General Practice and Primary Care, KCL School of Medicine, London SE116SP

roger.jones@kcl.ac.uk

Competing interests: None declared.

Provenance and peer review:

Commissioned; not externally peer reviewed.

BMJ 2007;334:704-5

doi: 10.1136/bmj.39170.410347.80
Coeliac disease affects around 1\% of the general population, but most cases are unrecognised and diagnosis is often delayed considerably. ${ }^{1}{ }^{2}$ This is surprising, given how common the disease is and how serious its effects can be. ${ }^{1}{ }^{4}$ Several possible reasons exist for this delay; the most important is that most patients with coeliac disease do not have typical symptoms of malabsorption. Even if these symptoms are present their non-specific nature may not trigger diagnostic suspicion of coeliac disease. Other atypical presentations can occur, especially in older patients, ${ }^{5}$ and the disease may even be seen in obese people. ${ }^{6}$

In this week's BMJ, Hopper and colleagues report a validated clinical prediction rule to determine an effective diagnostic method of detecting all cases of coeliac disease in people referred for gastroscopy. People with positive tissue transglutaminase antibodies and "low risk symptoms," as well as those with the high risk symptoms of diarrhoea, weight loss, and anaemia, were investigated with duodenal biopsy, while those with a negative antibody result and low risk symptoms were not biopsied. ${ }^{7}$ They found that pre-endoscopy serological testing combined with biopsy of people with high risk symptoms had a sensitivity of $100 \%$. They also found that a proportion of high risk patients with positive serology turn out not to have coeliac disease on biopsy, which should prompt reconsideration of the need for a lifelong gluten-free diet. An accompanying commentary in this issue by Graber and Kumar discusses the potential application of the decision rule to clinical practice. ${ }^{8}$

Coeliac disease is characterised by a lifelong intolerance to certain storage proteins contained in wheat, rye, and barley, collectively known as gluten, and it is an unusual combination of food intolerance and autoimmunity. Chronic inflammation of proximal small intestinal mucosa, with atrophy of small intestinal villi, is associated with impaired absorption of nutrients and increased secretion of water and solids because of abnormal intestinal permeability. The disease has been classified into four phenotypes. ${ }^{2}$ In classic coeliac disease, patients have intestinal malabsorption and gastrointestinal symptoms, whereas the atypical (most common) form of the disease has few or no gastrointestinal symptoms. Atypical disease has other problems, however, including iron deficiency anaemia, osteoporosis, short stature, infertility, and unfavourable outcomes of pregnancy. ${ }^{9}$ In silent coeliac disease, asymptomatic patients are found to have gluten induced villous atrophy, and in latent disease, patients may have normal mucosa or may show villous atrophy, which improves after gluten withdrawal.

The gold standard for the diagnosis of coeliac disease is a proximal small intestinal (duodenal) biopsy, but serum testing for antigliadin and endomysial antibodies is also available and offers reasonable sensitivity and specificity. The American Gastroenterology Association recommends the use, in primary care, of the IgA tissue 
transglutaminase antibody test as the single diagnostic serological test. ${ }^{2}$ However, coeliac disease may cause IgA deficiency, so that IgG endomysial antibodies and transglutaminase antibodies should be checked if biopsy is positive but the $\operatorname{IgA}$ tests are negative. Furthermore, because the histology of the disease can be patchy, careful quadrantic biopsies from the duodenum are needed. Even then difficulties in the preparation and interpretation of histological material may create diagnostic uncertainty, so that follow-up and reinvestigation may be needed to confirm the diagnosis. ${ }^{10}$

Coeliac disease is more common in people whose first degree relatives have the condition and in patients with iron deficiency anaemia, low bone mineral density, and other autoimmune disorders, such as type 1 diabetes mellitus and autoimmune thyroid and liver disease. Associations between coeliac disease and several other conditions including Down's syndrome, Turner's syndrome, and schizophrenia have been reported. The possible excess of coeliac disease in patients with irritable bowel syndrome and the need to test for the condition in the routine investigation of intermittent abdominal pain and bloating remain controversial. In a recent treatment trial of irritable bowel syndrome in the United Kingdom the prevalence of coeliac disease was $0.7 \%$, similar to the population mean. ${ }^{11}$ Coeliac disease is associated with excess mortality, including an increased risk of nonHodgkin's lymphoma and other cancers. ${ }^{12}$

A gluten-free diet not only protects against non-
Hodgkin's lymphoma, corrects anaemia, and restores normal nutritional and biochemical status, but it also substantially improves quality of life, particularly if troublesome gastrointestinal symptoms have been present. $^{13}$

What then are the important messages for primary care? Firstly, patients with unexplained diarrhoea, anaemia, weight loss, infertility, recurrent miscarriage and low birthweight babies, or low bone mineral density are at increased risk of coeliac disease and should be investigated in primary care with antibody tests. Secondly, risk of coeliac disease is higher in patients with a first degree relative with the condition and in those with other autoimmune disorders such as type 1 diabetes (with which coeliac disease sometimes shares the HLA markers DQ2 and DQ8) and autoimmune thyroid and liver disease, and serum antibody testing should be considered. Patients with gastrointestinal symptoms strongly suggestive of coeliac disease who test negative should be referred for a specialist opinion. In all patients with positive antibodies the diagnosis of coeliac disease must be confirmed by endoscopic biopsy, for which specialist referral will also usually be required. Thirdly, while a lifelong gluten-free diet can reverse the effects of gluten enteropathy, the diagnosis, once again, must be confirmed by a small intestinal biopsy. Hopper and colleagues' study offers an attractive algorithmic approach to identifying coeliac disease, and evaluation of this algorithm in a primary care setting is now required.

\section{The 2006 WHO child growth standards}

\section{Have implications for nutrition programmes in emergencies}

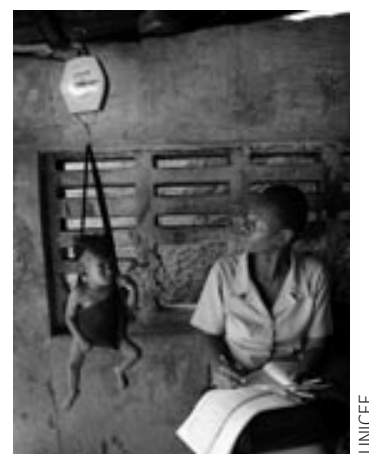

RESEARCH p 733

Martin Bloem chief of nutrition World Food Program, Rome 00148, Italy

Martin.Bloem@wfp.org

Competing interests: None declared. Provenance and peer review:

Commissioned; not externally peer reviewed.

BMJ 2007;334:705-6 doi: 10.1136/bmj.39155.658843.BE
In April 2006, the World Health Organization released its new WHO child growth standards, ${ }^{1} 16$ years after a WHO working group on infant growth recommended that these standards should describe how children should grow rather than how they actually grow. ${ }^{2}$ The basis for the new growth standards was six population based studies of infants and children from Ghana, India, Norway, Brazil, Oman, and North America, undertaken between 1997 and 2003. Participants were fed according to accepted international nutritional standards (including breast feeding), and their mothers were adequately nourished and avoided known adverse factors such as tobacco exposure.

The new growth standards show that children born in different regions of the world can and should grow equally well, and they also show that sex and ethnic origin are minor determinants of growth compared with adequate nutrition, environment, and health. ${ }^{2}$ However, as expected, important differences in the diagnosis of malnutrition emerge when the standard cut-offs are applied using either the National Center for Health Statistics (NCHS)-WHO reference or the WHO 2006 growth standards.

In this week's $B M J$, Seal and Kerac report the implications of adopting the new WHO child growth stand- ards in emergency and non-emergency child feeding programmes using secondary data analysis from three nutritional surveys in emergency settings. ${ }^{3}$ Nutritional status can be expressed using either $\mathrm{z}$ scores or percentage of the median values. WHO recommends that weight for height should be expressed as a $\mathrm{z}$ score. However, while not recommended by WHO, many agencies working in emergency settings use weight for height expressed as a percentage of the median as the criterion for admission to feeding programmes.

Seal and Kerac tabulated and compared the weight for height $\mathrm{z}$ score and percentage of the median cut-offs for moderate and severe acute malnutrition from both the NCHS-WHO growth reference and the new WHO standards. With the new WHO standards, a marked increase $(0.5-2.7)$ in the prevalence of severe wasting was seen if weight for height was expressed as a $\mathrm{z}$ score (weight for height $\leq 3 \mathrm{z}$ scores), confirming a previous report that showed an increase of 1.5-2.5. ${ }^{4}$ Paradoxically, however, the new WHO standards showed a significantly lower prevalence of severe wasting when expressed as a percentage of the median (weight for height $<70 \%$ of the median). These findings have serious programmatic and resource implications.

In emergencies, growth standards are used for 
various reasons, including interpreting the results of nutritional surveys, estimating potential beneficiary figures, and calculating entry and exit criteria for feeding programmes. Increased prevalence of severe wasting varies in different settings when using either the new WHO standards or the NCHS-WHO references. This fact makes the interpretation of nutritional surveys difficult and means that results cannot be converted from one to the other by using a simple algorithm. During the transition period, therefore, both the WHO standards and the NCHS-WHO references should be used until the implications of these differences are better understood.

The choice of using the percentage of the median or the $\mathrm{z}$ score for admission of children to feeding centres is more complicated and should be related to functional outcomes. Weight for height expressed as a percentage of the median is used partly because it is a better predictor of mortality than weight for height expressed as a $\mathrm{z}$ score when using the NCHS reference. ${ }^{5}$ We urgently need to study the functional significance of the weight for height indicators and investigate the suitable z score cut-offs for therapeutic and supplementary feeding in relation to mortality and other functional outcomes.

WHO, the United Nations World Food Programme, and Unicef are in the process of producing a joint statement on community based management of severe acute malnutrition. New evidence using ready to use foods suggests that large numbers of children with severe acute malnutrition can be treated in their communities without being admitted to therapeutic feeding centres or health facilities. ${ }^{6} 7$

It is difficult and costly for community health workers or volunteers to measure weight for height, and the mid-upper arm circumference is a good predictor of mortality. A mid-upper arm circumference less than $110 \mathrm{~mm}$ has therefore been recommended as an indicator of severe acute malnutrition in community based management of malnutrition. It would be useful to investigate whether this measurement can also be used as an indicator for admission to feeding programmes.

The international nutrition community working in emergencies has welcomed the introduction of the new WHO growth standard. Many will agree with Seal and Kerac, however, that a full assessment of the appropriate use of the new WHO standards in the diagnosis of acute malnutrition is urgently needed, and that this should be completed before they are adopted by agencies engaged in running nutritional programmes in emergencies.

\section{Intimate partner violence}

\section{Doctors' roles should be integrated with the needs of patients and society}

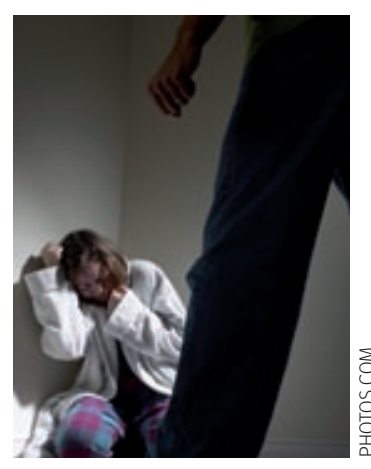

Lorraine E Ferris professor Public Health Sciences, Faculty of Medicine, University of Toronto, Toronto, ON, Canada M2K IZ4 Lorraine.ferris@utoronto.ca Competing interests: None declared. Provenance and peer review: Commissioned; not externally peer reviewed.

BMJ 2007;334:706-7 doi: 10.1136/bmj.39168.644757.BE
The World Health Organization's study on domestic violence against women highlights the need for immediate action. The study across 10 countries used robust culturally appropriate methods to assess the extent and effects on health of intimate partner violence and non-partner violence in 24000 women. The lifetime prevalence of physical or sexual intimate partner violence (or both) in women who had ever had a partner ranged from 15\% to $71 \%$ (29-62\% at most sites), though prevalence varied significantly between and within countries (large cities versus less populated areas).

We know more about the epidemiology of this type of violence than how to identify, prevent, and reduce it. However, recent research has made great strides, including identifying optimal methods for further evaluation of case screening in emergency departments, family medicine practices, and women's health clinics; examining women's acceptance of screening; identifying effective interventions; and identifying successful strategies for training and continuing medical education. Further research is still needed, though, especially to evaluate interventions and assess whether universal screening is effective.

We can learn much from WHO's methodology and data collection methods, which relied, among other things, on partnerships with women's organisations and with other key stakeholders within each country. Of the 15 recommendations in the $\mathrm{WHO}$ report, two concern strengthening the health sector response (box 1) and take a clinical and public policy perspective, as others have done. The recommendations inform the basis for defining the competency of doctors when dealing with intimate partner violence. If the identified competencies reflect the needs of patients, and, as much as possible, broader needs of society, then we are a small step closer to implementing these two WHO recommendations.

The CanMEDS (Canadian medical education directives for specialists) framework on the competency of doctors, which was developed by the Royal College of Physicians and Surgeons of Canada, is currently used in several countries. It is intended for use by educators, teachers, trainees, practising doctors, researchers, and other health professionals (for example, for understanding how doctors work on teams). Although CanMEDS may not be completely transferable across specialties and jurisdictions or valid for non-specialists, it is useful to consider what constitutes competency when dealing with intimate partner violence.

CanMEDS recognises seven (overlapping) roles for doctors-medical expert (central role), communicator, collaborator, health advocate, manager, scholar, and professional (box 2). Each role has its own elements and key enabling competencies that have been described in detail elsewhere. Box 3 gives examples of how the CanMEDS roles can help direct doctors towards practising effectively when dealing with intimate partner violence.

While CanMEDS has been used here for illustrative 
Box 1 Recommendations from the WHO study ${ }^{1}$ aimed at strengthening the health sector response Develop a comprehensive health sector response to the effects of violence against women Identify roles for health professionals in advocating for prevention of violence and in providing services to women

Coordinate and work with other health professionals and with other sectors that care for abused women (for example, by creating formal referral processes and protocols)

Integrate appropriate non-stigmatising, non-blaming, respectful, secure, and confidential responses to violence against women into:

All aspects of care (such as emergency services, reproductive health services, mental

health services, HIV related services)

Sensitising and training of health professionals

Use reproductive health services as entry points for identifying and supporting women in abusive relationships, and for delivering referral or support services

Sensitise and train reproductive health providers to recognise and respond appropriately to violence by having protocols to deal with it, using referral systems (if such systems are unavailable, providing information about legal and counselling options), ensuring confidentiality, and making women's safety a priority

Add an anti-violence component to antenatal services, parenting classes, and other services that involve men

Box 2 CanMEDS definitions of doctors' roles. Reprinted with permission from appendix B of the CanMEDS 2005 physician competency framework ${ }^{9}$

\section{Medical expert (the central role)}

As Medical experts, physicians integrate all of the CanMEDS roles, applying medical knowledge, clinical skills, and professional attitudes in their provision of patient-centered care

\section{Communicator}

As Communicators, physicians effectively facilitate the doctor-patient relationship and the dynamic exchanges that occur before, during, and after the medical encounter

Collaborator

As Collaborators, physicians effectively work within a healthcare team to achieve optimal patient care Manager

As Managers, physicians are integral participants in healthcare organizations, organizing sustainable practices, making decisions about allocating resources, and contributing to the effectiveness of the healthcare system

Health advocate

As Health advocates, physicians responsibly use their expertise and influence to advance the health and well-being of individual patients, communities, and populations

Scholar

As Scholars, physicians demonstrate a lifelong commitment to reflective learning, as well as the creation, dissemination, application and translation of medical knowledge

Professional

As Professionals, physicians are committed to the health and well-being of individuals and society through ethical practice, profession-led regulation, and high personal standards of behaviour

purposes, other competency frameworks could also be used. The concept of developing doctors' competency in intimate partner violence is not new. To be valuable, these frameworks need to share the CanMEDS focus on what distinguishes doctors from other health professionals (medical expert) and on competencies that go beyond doctors' technical knowledge and skills to the needs and expectations that society places upon them. An open debate about what constitutes such competencies is needed to develop an international perspective and to guide training programmes and continuing medical education. We need to learn from educational leaders about their experiences and evaluations of providing training about intimate partner violence using learning goals matched to competencies. We also need to find effective ways to deal with the resistance that may come from adding this to medical school curriculums.
Box 3 Key features of the health sector WHO recommendations, ${ }^{1}$ with examples of CanMEDS roles ${ }^{9}$ in dealing with intimate partner violence

Integrating appropriate responses to violence against women into all aspects of clinical care

Medical expert

Apply best practice to identify, intervene, and refer cases of intimate partnerviolence

Professional

Deliver ethical, humane and compassionate care, recognising that blame may be cast, which may cause secondary adverse effects. Recognise limitations in expertise (and seek consultation if necessary)

Communicator

Facilitate patient centred therapeutic communication, including active empathic listening to establish trust. Work therapeutically with victims of intimate partner violence and share decision making

Manager

Participate in health systems for collaborative decision making and quality improvement. Victims of intimate partner violence need health services; doctors must manage and improve the effectiveness of their individual provider and overall programme within healthcare systems

Scholar

Identify and apply evidence based intimate partner violence screening and interventions, identify knowledge or practice gaps and model these competencies for others

Advocating for prevention and for services Health advocate and medical expert

Use medical expertise and influence to improve the overall health of patients and populations. Identify and apply health determinants (social, cultural, economic) and strategies to promote health and prevent disease. Doctors must know how to help victims of intimate partner violence in navigating local systems and obtaining appropriate resources

Working with other health professionals and other sectors

Collaborator

Work effectively and appropriately within health and non-health sectors to facilitate coordinated intimate partner violence responses for individual patients and populations

Sensitising and training health professionals

Scholar

Educate patients and other providers about intimate partner violence; engage in lifelong learning through critical appraisal of evidence and evidence based practice

Professional

Serve as a role model; commit to appropriately dealing with intimate partnerviolence

We must recognise the difference between a doctor knowing and being able to perform a competency and actually implementing that competency in practice. Once we have international consensus on what constitutes such competency, the next step is to assess it in practice, and ultimately to use it to set standards and measure performance.

Finally, we must recognise that efforts to identify what constitutes competency of doctors in dealing with intimate partner violence must be considered in tandem with research, as evidence is still unavailable for many aspects of patient care (for example, universal screening versus diagnostic case-finding methods). All this can only be achieved if doctors and society acknowledge the epidemic of intimate partner violence, recognise it as a global health and human rights issue, and devote resources to dealing with it. 


\section{Hospital acquired infection}

\section{Control measures for Clostridium difficile need to extend into the community}

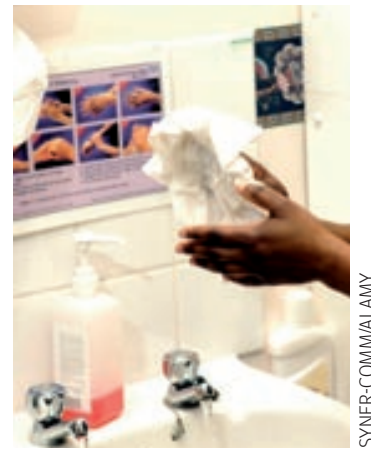

John Starr reader in geriatric medicine

University of Edinburgh, Royal

Victoria Hospital, Edinburgh

EH42DN

jstarr@staffmail.ed.ac.uk

Competing interests: None declared.

Provenance and peer review:

Commissioned; not externally peer reviewed.

BMJ 2007;334:708 doi: 10.1136/bmi.39169.601285.80
Recent data published by the Health Protection Agency (HPA) show that each year in England around 7000 inpatients have methicillin resistant Staphylococcus aureus (MRSA) bacteraemia and more than 50000 inpatients aged 65 years and over have Clostridium difficile infections. ${ }^{1} C$ difficile cases rose by $5.5 \%$ in 2006 compared with 2005 , whereas MRSA cases fell by $4.3 \%$ over a similar period. HPA spokespeople said they thought that the rate of increase of $C$ difficile was slowing and MRSA rates had reached a plateau.

My own time series analyses of the reported data failed to detect any significant change in rates of $C$ difficile or MRSA, though $C$ difficile increases significantly during winter (www.geriatric.med. ed.ac.uk/john_starr.htm). The seasonal variation may be a result of many older people who require antibiotic treatment being admitted to hospital at that time of year. Despite the HPA data, there is a consensus that hospital acquired infection rates remain high and that recent control measures are having only a limited effect, especially on $C$ difficile. With more than 2200 deaths attributed to $C$ difficile on death certificates in England and Wales in 2004, the mortality rate is fast approaching that for road traffic accidents and is now around half that for suicide. ${ }^{2}$

Control of $C$ difficile is difficult because, unlike MRSA, alcohol hand scrubs are ineffective and its spores are resistant to routine hospital cleaning. ${ }^{3}$ Moreover, old and frail patients are at highest risk of infection with $C$ difficile. Since older people are living longer, hospital admissions of people over 85 years have increased relative to other age groups. ${ }^{4}$ This continuing change in case mix is likely to increase the absolute number of reported cases.

In December 2006, the Department of Health issued a letter on healthcare associated infections, in particular infection caused by $C$ difficile, which called for urgent action. ${ }^{5}$ In addition to hand hygiene and environmental cleaning, recommendations include prudent antibiotic prescribing, isolation of infected patients, and use of personal protective equipment. Although there is a trend for reduced prescription of antibiotics in the community, it remains high in hospitals and accounts for $59 \%$ of prescription costs. ${ }^{6} 7$ Theoretically, isolation of infected patients should not be difficult. The National Health Service in England still has around 150000 beds and even if just 20\% of these are single rooms that can be used for isolation purposes, there should still be more than adequate capacity. However, the clustering of cases can put a strain on local resources. This is a particular concern with the emergence of hypervirulent strains. ${ }^{8}$

Another factor that may be driving the incidence of infection with $C$ difficile is the community reservoir. Carriage rates in healthy people in the community may be around $5 \%$, perhaps substantially higher in those connected with hospitals, and this may lead to community acquired infection. ${ }^{9}$ Indeed, the relative increase in community acquired $C$ difficile far outstrips that seen in hospital, despite reduced antibiotic use, and may relate to increased use of proton pump inhibitors and other drugs that suppress gastric acid production. ${ }^{10}$ More than 13000 cases of community acquired $C$ difficile occur each year in the UK, three quarters of which are in people who have not been in hospital during the previous year. In contrast, the HPA identified fewer than 100 community acquired cases of MRSA between 2003 and 2005. This raises the question of whether $C$ difficile can still be thought of as purely a hospital acquired infection and, if not, whether other infection control measures are needed, such as screening people in the community before they are admitted electively.

Early accurate diagnosis is fundamental to any infection control programme, whether based in hospital or the community. Laboratory methods to detect $C$ difficile have varied considerably in the UK. ${ }^{11}$ Though variation has been reduced in England, it is still difficult to make comparisons with data from other countries, and thus assess the effects of their infection control policies. Denmark, for example, preferred culture to toxin detection as a diagnostic tool. Denmark also reported two fatal cases of $C$ difficile enterocolitis in elephants in 2006, a reminder that animals, including household pets, can be a reservoir for the organism.

A report from the HPA published last year recommended greater international cooperation to tackle $C$ difficile by sharing information about the appearance of new strains or changes in the prevalence of known strains.

It also emphasised that infection control guidelines have stayed essentially the same for more than a decade, and were implemented inconsistently across England. ${ }^{12}$ The recent HPA report presents a mixed message. Although both $C$ difficile and MRSA are closely associated with use of antibiotics, in other ways they are quite different. Infection control policies for MRSA have been more successful than for $C$ difficile, yet data on hospital acquired infections are often grouped together. In particular, because of the rise in community acquired infection it is important to consider whether a $C$ difficile infection control policy solely focused on hospitals remains appropriate. 